\title{
Study on Contact Fatigue Crack Propagation Behavior of $\mathrm{Cr}_{7} \mathrm{C}_{3}$ Coatings
}

\author{
Wentong Cui ${ }^{1,2}$, a , Juan Feng ${ }^{1,2, b, ~}{ }^{*}$ and Ling Tian ${ }^{1,2, c}$ \\ ${ }^{1}$ Department of Mechanical Engineering, Tsinghua University, Beijing, 100084, China \\ ${ }^{2}$ Beijing Key Lab of Precision/Ultra-precision Manufacturing Equipment and Control, Tsinghua \\ University, Beijing, 100084, China
}

accuiwt14@mails.tsinghua.edu.cn, bfengj@tsinghua.edu.cn, ctianling@mail.tsinghua.edu.cn

\begin{abstract}
Keywords: Contact Fatigue, Crack Propagation, Overrunning Clutch, $\mathrm{Cr}_{7} \mathrm{C}_{3}$ Coatings, XFEM.
\end{abstract}
\begin{abstract}
In this paper, the contact fatigue crack propagation behavior of $\mathrm{Cr}_{7} \mathrm{C}_{3}$ coatings was studied by means of extended finite element method and linear elastic fracture mechanics. The contact fatigue model of simplified geometric model was established by commercial software ABAQUS. The influence of the thickness of coatings, the elastic modulus of the coatings, the elastic modulus ratio of the coatings and the substrate on the contact fatigue life of the coatings was studied. The optimal coatings design parameters were obtained.
\end{abstract}

\section{Introduction}

A Positive continuous engagement (PCE) overrunning clutch is the essential component of helicopter transmission system. It is a critical element to ensure the correct combination and disengaged of the main reducer from the engine. A PCE overrunning clutch is composed of wedges, cages and a spring. Its function is to transfer the torque of the output shaft of the engine to the input shaft of the main reducer. Using chemical vapor deposition (CVD) method to plate $\mathrm{Cr}_{7} \mathrm{C}_{3}$ coatings on the surface of wedges aims to reduce the wear of wedges. During the flight of helicopter, we found that the actual life of the coatings is much less than the design life of the coatings.

According to the stress analysis, it can be seen that the wedges are subjected to cyclic normal pressure and alternating tangential sliding friction force. The surface of the coatings was observed by scanning electron microscope (SEM). After the analysis, it can be concluded that the mechanism of the fatigue spallation of $\mathrm{Cr}_{7} \mathrm{C}_{3}$ coatings is as follows. At first, the distribution of shear stress in the coatings will occur under the action of alternating contact load. The location of the maximum shear stress is the weak position of the coatings, and the micro cracks or micro defects at this location are the sources of fatigue crack initiation. Then, the cracks will extend in the direction of 45 degrees under the maximum shear stress. In the process of crack propagation, the crack is fused with other micro cracks, and finally extends to the coatings' surface. Finally, the coatings spalled under the action of the surface force.

In order to improve the fatigue life of the coatings, the influence of coatings parameters on the crack propagation behavior of the coatings is studied. Extended finite element method (XFEM) can simulate crack propagation effectively $[1,2]$. Sukumar uses the XFEM and the fast stepping method to simulate the 3D fatigue crack propagation, and uses the fast stepping method and the Paris formula to simulate the crack tip movement [3]. Baydoun studies of the reliability of different crack propagation criterion under the XFEM and 3D fracture mechanics method, concludes that the maximum energy release rate criterion and the maximum circumferential tensile stress criterion can be used to accurately describe the crack propagation process [4, 5]. Baietto combines the experimental results and the XFEM to simulate 2D and 3D contact fatigue crack growth [6].

In this paper, the XFEM in ABAQUS and Paris formula are used to study the effects of coatings thickness, coatings elastic modulus, elastic modulus ratio of coatings and substrate, magnitude of load and loading frequency on the fatigue crack propagation behavior. 


\section{Extended finite element method and Paris formula}

When the traditional finite element method is used to simulate the static discontinuity problems such as cracks, the mesh partition needs to be consistent with the geometric discontinuity. The mesh near the crack tip needs to be refined. When the crack propagation is simulated, the gird needs to be updated with crack propagation.

The XFEM is used to solve the problem that the mesh needs to be updated in real time. In 1996, Melenk and Babuska put forward the method of unit decomposition, so that the local enrichment function can be applied to the finite element method [7]. In 1999, Belyschko and Black proposed an extended finite element method based on the unit decomposition method to describe the discontinuity of the crack with the combination of additional degrees of freedom and special enrichment functions [1].

When the crack propagation is simulated, the XFEM based on linear elastic fracture mechanics (LEFM) can be used. According to the theory of LEFM, the strain energy release rate of crack propagation is equal to that of crack closure. The criterion of crack initiation and propagation is determined by Pairs law when simulating low cycle fatigue crack [8].

As shown in Fig. 1, the crack growth rate region which in line with Paris law has two critical values, $G_{\text {thresh }}$ and $G_{p l}$. When the energy release rate $G$ is less than $G_{\text {thresh }}$, the crack does not initiate or expand. When the energy release rate is greater than $G_{p l}$, the crack growth rate increases exponentially. $G_{C}$ is the critical energy release rate.

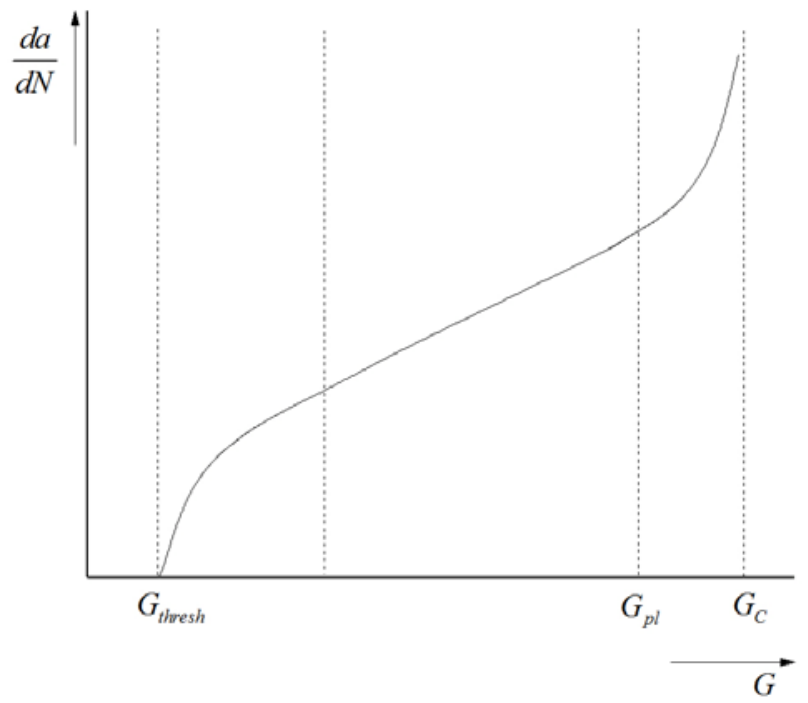

Fig. 1 Two or more references

When using Paris law [9] to determine crack initiation, the following two criteria must be met:

1) The maximum crack energy release rate within a period, $G_{\max }$ is greater than $G_{\text {thresh }}$;

2) $f=\frac{N}{c_{1} \Delta G^{c_{2}}} \geq 1.0$

In the formula, $c_{1}$ and $c_{2}$ are material constants. $N$ is the number of cycles. $\Delta G$ is the difference between the maximum crack energy release rate and the minimum crack energy release rate.

After crack initiation, crack growth rate, $\frac{d a}{d N}$, is determined by the relative crack energy release rate, $\Delta G$. The formula is:

$$
\frac{d a}{d N}=c_{3} \Delta G^{c_{4}}
$$

In the formula, $c_{3}$ and $c_{4}$ are material constants. The boundaries of validity of this formula is $G_{\text {thresh }}<G_{\max }<G_{p l}$. 


\section{Settings of simulation parameters}

The geometric model used in this paper is the side length of $1 \mathrm{~mm}$ cube, one of the surface is covered by $\mathrm{Cr}_{7} \mathrm{C}_{3}$ coatings of $0.008 \mathrm{~mm}$ thickness. Study on low cycle fatigue crack propagation behavior of $\mathrm{Cr}_{7} \mathrm{C}_{3}$ coatings by using the XFEM based on LEFM. The combination of the substrate and the coatings is tie bound.

The elastic modulus, tensile strength and Poisson's ratio of $\mathrm{Cr}_{7} \mathrm{C}_{3}$ and $\mathrm{GCr} 15$ is shown in Table 1. GCr15 is the substrate material.

I type, II type, III type critical crack energy release rates of $\mathrm{Cr}_{7} \mathrm{C}_{3}, G_{I C}, G_{I I C}, G_{I I I C}$ are $4.22 \times 10^{-4} \mathrm{MPa} \cdot \mathrm{m}$. Power low parameters, $a_{m}, a_{n}, a_{o}$, are 1.0. The material constants of the coatings material are $c_{1}=0.5, c_{2}=-0.1, c_{3}=4.8768 \times 10^{-8}$ and $c_{4}=2.1625$. The threshold of energy release rate for crack initiation and propagation is $\frac{G_{\text {thresh }}}{G_{C}}=0.001$. The upper limit of energy release rate for Paris law is $\frac{G_{p l}}{G_{C}}=0.85$.

Table 1 Material properties of $\mathrm{Cr}_{7} \mathrm{C}_{3}, \mathrm{GCr} 15$

\begin{tabular}{|c|c|c|c|}
\hline $\begin{array}{c}\text { Materia } \\
1\end{array}$ & Young's modulus $(\mathrm{GPa})$ & Poisson's ratio & Tensile strength $(\mathrm{MPa})$ \\
\hline $\mathrm{Cr}_{7} \mathrm{C}_{3}$ & 214.925 & 0.38 & 2500 \\
\hline $\mathrm{GCr} 15$ & 210.15 & 0.2887 & - \\
\hline
\end{tabular}

In ABAQUS, the bottom of the substrate is completely fixed. According to the Hertz contact theory [10], the half width of the contact surface, $b$, is $0.26484 \mathrm{~mm}$, the maximum contact stress, $\sigma_{\max }$, is $2967 \mathrm{MPa}$, because the coatings is subjected to normal pressure and tangential friction force. According to the Johnson formula [11], the normal load distribution is:

$$
P(x)=2967 \sqrt{1-14.2 x^{2}}
$$

When the friction coefficient, $\mu$, is 0.18 , the tangential load distribution can be obtained:

$$
Q(x)=534 \sqrt{1-14.2 x^{2}}
$$

The coatings are subjected to alternating loads and the loading frequency is $1 \mathrm{~Hz}$. Loading mode is linear loading, linear unloading. In ABAQUS, using the grid type of C3D8R to mesh the model. The grid type, C3D8R, is three-dimensional 8 nodes hexahedral elements.

\section{Influence of Coatings Parameters on Contact Fatigue Crack Propagation}

\subsection{The Thickness of $\mathrm{Cr}_{7} \mathrm{C}_{3}$ Coatings}

In order to study the influence of coatings thickness on contact fatigue life, a series of different coatings thickness values were simulated, as shown in Table 2. The other parameters and loading conditions in the simulation process are unchanged, that is, the elastic modulus of the coatings is $302.3 \mathrm{GPa}$ and the modulus of the substrate is $210.15 \mathrm{GPa}$.

Table 2 The list of coatings thickness

\begin{tabular}{|c|c|c|c|c|c|c|c|}
\hline Serial Number & 1 & 2 & 3 & 4 & 5 & 6 & 7 \\
\hline $\begin{array}{c}\text { Thickness }(\mu \mathrm{m} \\
)\end{array}$ & 4 & 8 & 12 & 16 & 20 & 24 & 28 \\
\hline
\end{tabular}

The number of crack expansion elements and the thickness of the coatings curve is shown in Fig. 2. It can be seen from the figure that when the coatings thickness is thin, the number of crack expansion elements is larger and the coatings life is smaller. With the increase of coatings thickness, the contact fatigue life increases and then decreases when the coatings thickness is 16 microns, the contact fatigue life of the coatings is the longest. When the coatings thickness is increased to 23 microns, the contact fatigue life of the coatings is basically the same as the coatings thickness increases. The 
relationship between the maximum principal stress and the thickness of the coatings is shown in Fig. 3. The relationship between the maximum principal stress and the thickness of the coatings and the relationship between the number of crack expansion elements and the thickness of the coatings is basically the same, which confirms the simulation results.

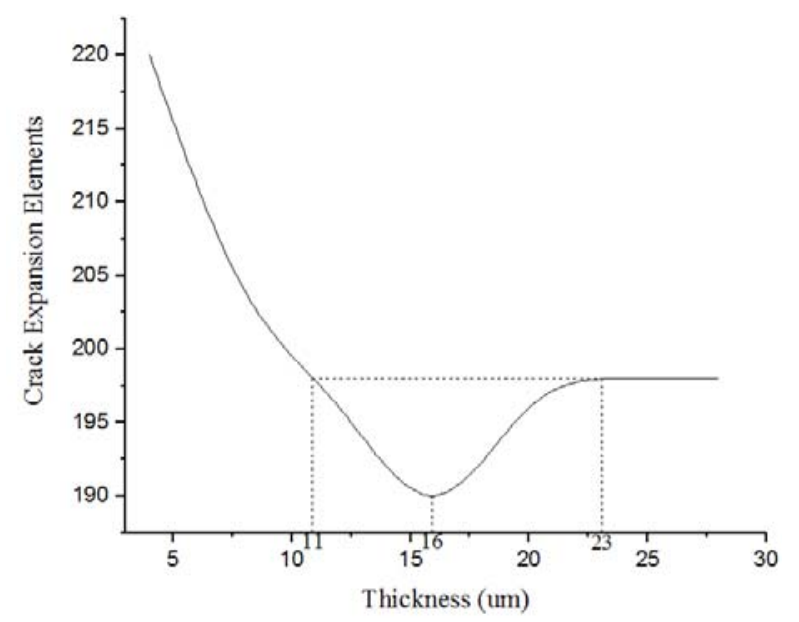

Fig. 2 The relationship between number of crack expansion elements and thickness of the coatings

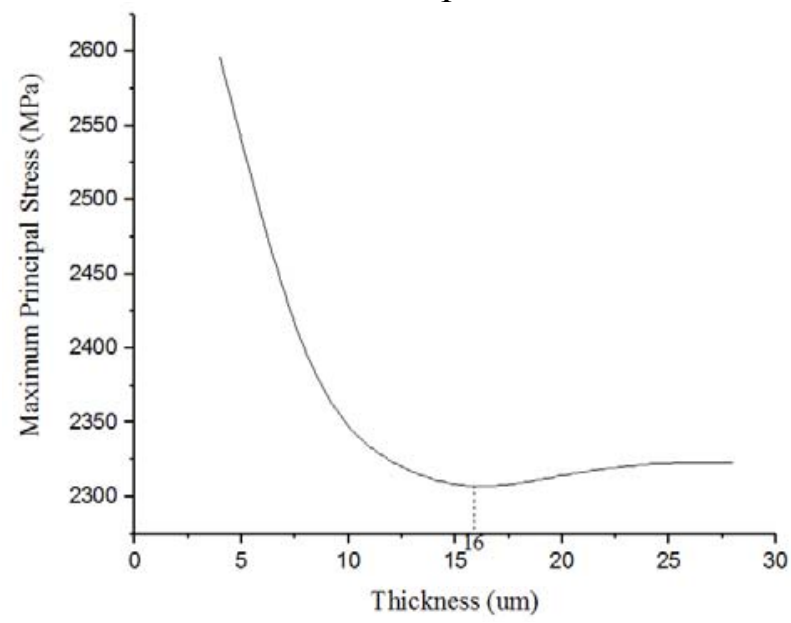

Fig. 3 The relationship between maximum principal stress and thickness of the coatings

The maximum principal stress curve of the cross section of different coatings thickness is shown in Fig. 4. It can be seen from the figure that when the coatings thickness is between 4 and 16 microns, the maximum principal stress value is obtained in the coatings, and when the coatings thickness is greater than 16 microns, the maximum principal stress value is obtained between the coatings and the substrate junction.

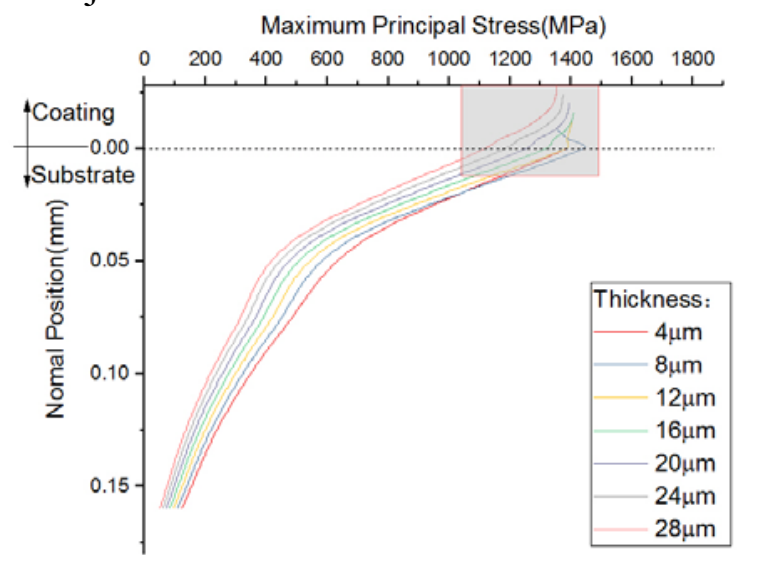

(a) Overall curve

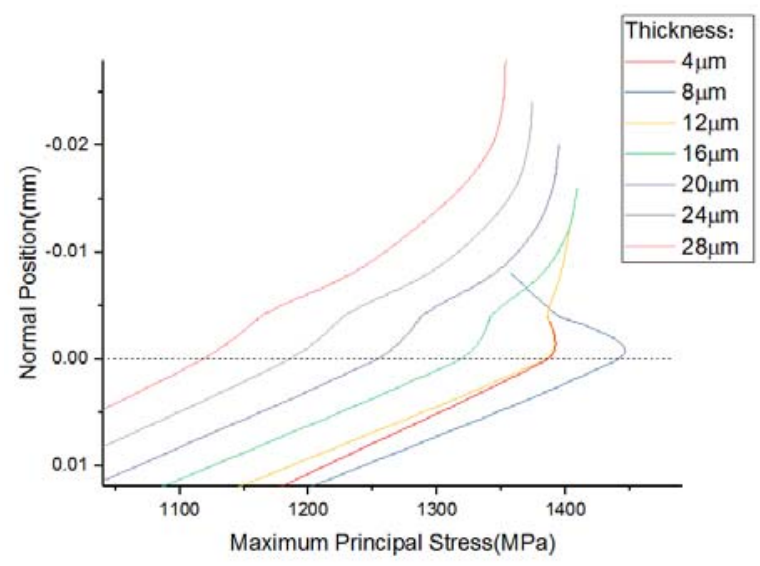

(b) Partial magnification

Fig. 4 The maximum principal stress curve of the cross section of different coatings thickness

In summary, when the coatings thickness is greater than 16 microns, the crack is easy to expand and the shear stress inside the coatings is large. Coating contact fatigue life is low. So the coatings 
thickness cannot be greater than 16 microns. At the same time, the coatings thickness cannot be too low, too low when the crack easy to expand. Therefore, when the elastic modulus of the coatings is $302.3 \mathrm{GPa}$ and the elastic modulus of the substrate is $210.15 \mathrm{GPa}$, the coatings thickness is preferably in the range of 11 microns to 16 microns.

\subsection{The Elastic Modulus of $\mathrm{Cr}_{7} \mathrm{C}_{3}$ Coatings}

In order to study the influence of the elastic modulus of the coatings on the fatigue life of the contact, the simulation of the elastic modulus of a series of different coatings is shown in Table 3 . Other parameters and loading conditions in the simulation process are unchanged, that is, the coatings thickness is 8 microns, and the substrate elastic modulus is $210.15 \mathrm{GPa}$.

Table 3 The list of the elastic modulus of the coatings

\begin{tabular}{|c|c|c|c|c|c|c|c|c|c|c|}
\hline Serial Number & 1 & 2 & 3 & 4 & 5 & 6 & 7 & 8 & 9 & 10 \\
\hline Elastic Modulus(GPa) & 100 & 150 & 200 & 250 & 350 & 400 & 450 & 500 & 550 & 600 \\
\hline
\end{tabular}

The simulation results of the elastic modulus of different coatings are simulated and the results are shown in Fig. 5. It can be seen from the figure that with the increase of the elastic modulus of the coatings, the number of crack propagation increases gradually, which indicates that the contact fatigue life of the coatings is shortened gradually. From the simulation results, the smaller the elastic modulus of the coatings, the longer the contact fatigue life of the coatings. The relationship between the maximum principal stress and the elastic modulus of the coatings is shown in Fig. 6 . The relationship between the maximum principal stress and the elastic modulus of the coatings and the relationship between the number of crack expansion elements and the elastic modulus of the coatings is basically the same, which confirms the simulation results.

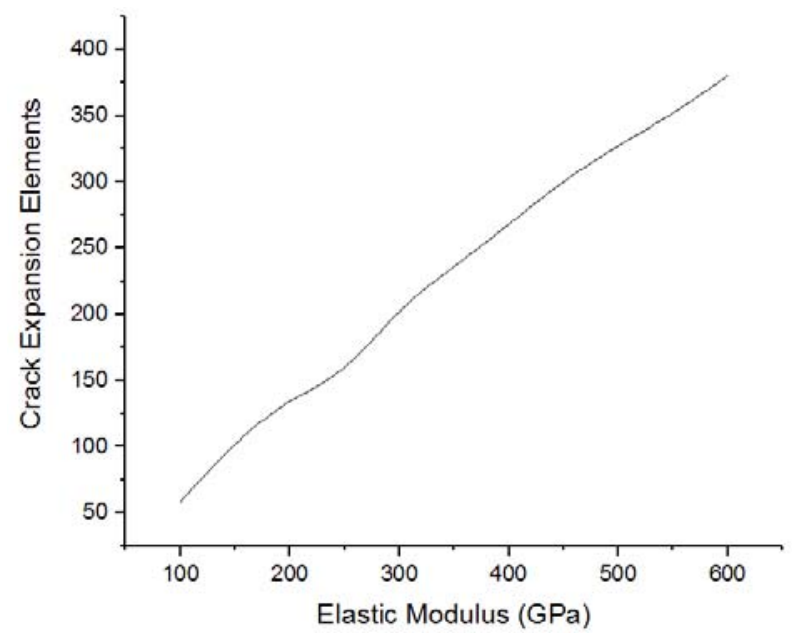

Fig. 5 The relationship between number of crack expansion elements and elastic modulus of the coatings

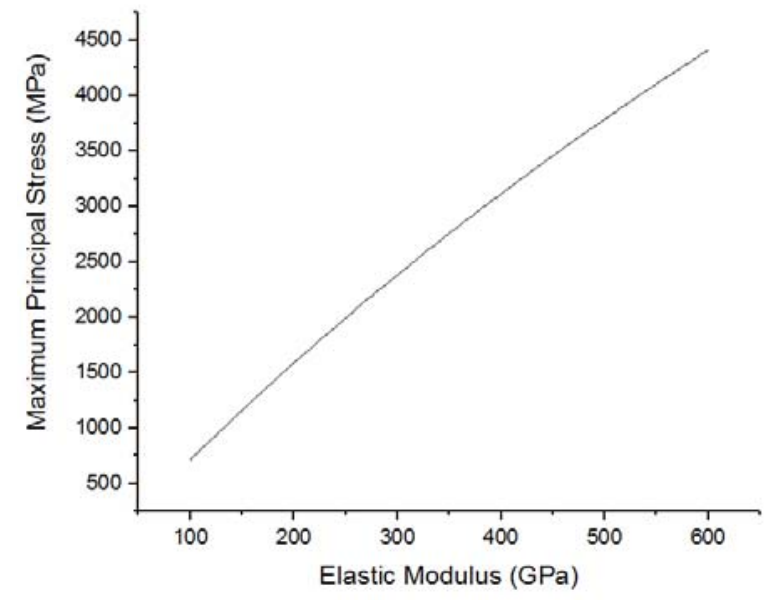

Fig. 6 The relationship between maximum principal stress and elastic modulus of the coatings 
The maximum principal stress curve of the cross section of different elastic modulus of the coatings is shown in Fig. 7. It can be seen from the figure that when the elastic modulus of the coatings is $1 \mathrm{GPa}$ and $1.5 \mathrm{GPa}$, the maximum principal stress is in the substrate, and the stress in the coatings is small. When the elastic modulus of the coatings is between $2 \mathrm{GPa}$ and $6 \mathrm{GPa}$, the position of the maximum principal stress appears at the interface between the coatings and the substrate, and as the elastic modulus increases, the value of the maximum principal stress increases gradually.

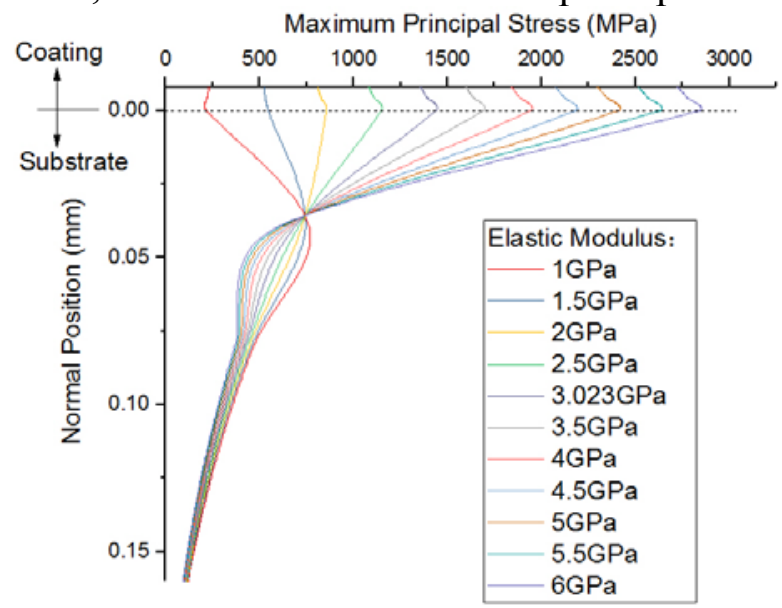

Fig. 7 The maximum principal stress curve of the cross section of different elastic modulus of the coatings

Based on the above analysis, the elastic modulus of the coatings should be as small as possible when the coatings thickness is 8 microns and the substrate elastic modulus is $210.15 \mathrm{GPa}$, considering only the contact fatigue failure. And the optimum value is between $1 \mathrm{GPa}$ and $1.5 \mathrm{GPa}$.

\subsection{The Elastic Modulus Ratio between $\mathrm{Cr}_{7} \mathrm{C}_{3}$ Coatings and Substrate Material}

In order to study the influence of the elastic modulus ratio of the coatings and the substrate on the contact fatigue life, a series of different coatings and substrate elastic modulus ratio were simulated, as shown in Table 4. Other parameters and loading conditions in the simulation process are unchanged, the coatings thickness is 8 microns.

Table 4 The list of the elastic modulus ratio of the coatings and the substrate

\begin{tabular}{|c|c|c|c|c|c|c|c|c|c|}
\hline Serial Number & 1 & 2 & 3 & 4 & 5 & 6 & 7 & 8 & 9 \\
\hline Elastic Modulus Ratio & $9: 1$ & $9: 2$ & $9: 3$ & $9: 4$ & $9: 5$ & $9: 7$ & $9: 8$ & $9: 9$ & $9: 10$ \\
\hline
\end{tabular}

The simulation results of different coatings and substrate elastic modulus ratio are simulated and calculated. The results are shown in Fig. 8. The results show that the larger the elastic modulus ratio of the coatings and the substrate, the greater the rate of contact fatigue crack growth in the coatings. According to the simulation results, when the coatings and the substrate are selected, the elastic modulus of the coatings should be as close as possible to the elastic modulus of the substrate when the material meets the other requirements. The relationship between the maximum principal stress and the elastic modulus ratio at the crack is shown in Fig. 9. The relationship between the maximum principal stress and the elastic modulus ratio at the crack and the relationship between the number of crack expansion elements and the elastic modulus ratio of the coatings and substrate is basically the same, which confirms the simulation results. 


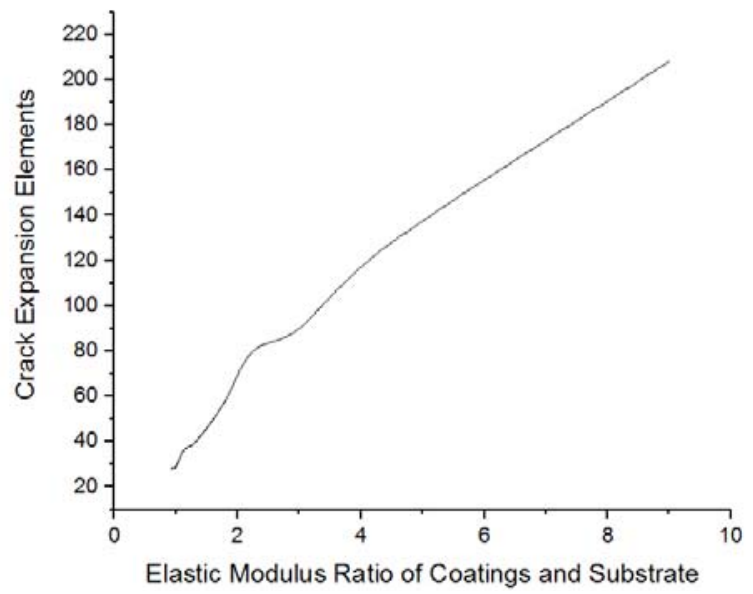

Fig. 8 The relationship between number of crack expansion elements and elastic modulus ratio of the coatings and the substrate

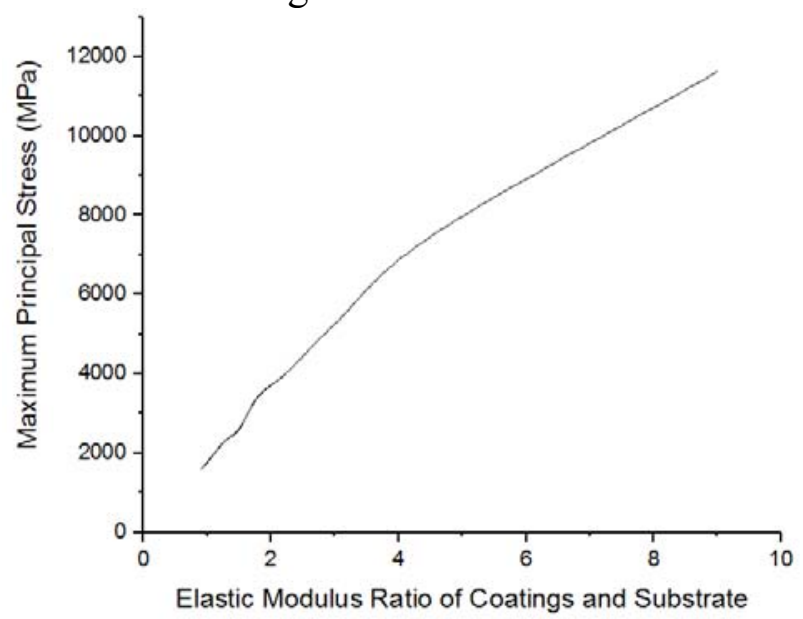

Fig. 9 The relationship between maximum principal stress and elastic modulus ratio of the coatings and the substrate

The maximum principal stress curve of the cross section of different elastic modulus ratio of the coatings and substrate is shown in Fig. 10. It can be seen from the figure that when the elastic modulus ratio of the coatings and the substrate is relatively large, the internal stress of the coatings reaches about $8000 \mathrm{MPa}$, and the coatings will expand rapidly and then peel off under the stress. When the elastic modulus ratio between the coatings and the substrate is less than 1.5, the internal stress of the coatings is between $1000 \mathrm{MPa}$ and $2000 \mathrm{MPa}$, and the maximum principal stress appears in the substrate, and the fatigue crack growth rate in the coatings is low.

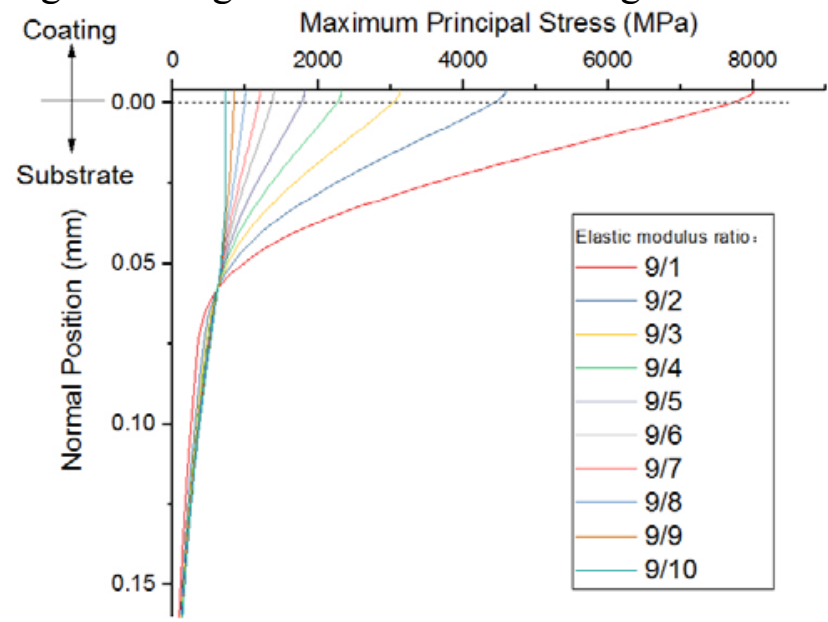

Fig. 10 The maximum principal stress curve of the cross section of different elastic modulus ratio of the coatings and the substrate 
In the above analysis process, when the coatings thickness is 8 microns, the elastic modulus of the coatings should be as close as possible to the substrate material, the elastic modulus ratio of the coatings and the substrate material should not exceed 1.5.

\section{Conclusion}

In this paper, the contact fatigue crack propagation model of $\mathrm{Cr}_{7} \mathrm{C}_{3}$ coatings was established by commercial software ABAQUS, and the influence of coatings thickness, elastic modulus of coatings and elastic modulus ratio of coatings and substrate on the contact fatigue life of coatings was studied.

When the elastic modulus of the coatings is $302.3 \mathrm{GPa}$ and the elastic modulus of substrate material is $210.15 \mathrm{GPa}$, the coatings thickness should be in the range of 11 microns to 16 microns. When the coatings thickness is 8 microns and the elastic modulus of the substrate material is $210.15 \mathrm{GPa}$, the elastic modulus of the coatings should be as small as possible and the optimum value is between $1 \mathrm{GPa}$ and $1.5 \mathrm{GPa}$. When the coatings thickness is 8 microns, the elastic modulus of the coatings should be as close as possible to the substrate material, the elastic modulus ratio of the coatings and the substrate material should not exceed 1.5.

\section{Acknowledgments}

This work was financially supported by National Natural Science Foundation of China (Grant No.51175287).

\section{References}

[1] T. Belytschko, T. Black, Elastic crack growth in finite elements with minimal remeshing, Int. J. Numer. Meth. Eng. 45 (1999) 601-620

[2] J. Dolbow, T. Belytschko, A finite element method for crack growth without remeshing, Int. J. Numer. Meth. Eng. 46 (1999) 131-150

[3] N. Sukumar, D. L. Chopp, B. Moran, Extended finite element method and fast marching method for three-dimensional fatigue crack propagation, Eng. Fract. Mech. 70 (2003) 29-48

[4] M. Baydoun, T. P. Fries, Crack propagation criteria in three dimensions using the XFEM and an explicit-implicit crack description, Int. J. Fract. 178 (2012) 51-70

[5] T. P. Fries, M. Baydoun, Crack propagation with the extended finite element method and a hybrid explicit-implicit crack description, Int. J. Numer. Meth. Eng. 89 (2012) 1527-1558.

[6] M. C. Baietto, E. Pierres, A. Gravouil, et al, Fretting fatigue crack growth simulation based on a combined experimental and XFEM strategy, Int. J. Fatigue. 47 (2013) 31-43.

[7] M. L. Benzeggagh, M. Kenane, Measurement of mixed-mode delamination fracture toughness of unidirectional glass/epoxy composites with mixed-mode bending apparatus, Comp. Sci. Technol. 56 (1996) 439-449

[8] Abaqus 6.14 HTML Documentation/Documentation/Documentation Abaqus Analysis User's Guide, Dassault Systèmes Simulia Corp

[9] E. M. Wu, R. C. Reuter, Crack extension in fiberglass reinforced plastics and a critical examination of the general fracture criterion, TAM Report. (1965) 275

[10] P. C. Paris, M. P. Gomez, W. E. Anderson, A rational analytic theory of fatigue, The trend in engineering. 13 (1961) 9-14

[11] H. Hertz, On the contact of elastic solids, J. reine angew. Math. 92 (1881) 156-171

[12] K. L. Johnson, Contact mechanics, Cambridge university press. 1987, chapter 3 